\title{
ON THE STRUCTURE OF ALGEBRAIC ALGEBRAS AND RELATED RINGS
}

\author{
BY \\ JAKOB LEVITZKI
}

1. Introduction. The systematic study of algebraic algebras has been initiated by N. Jacobson [6] $\left(^{1}\right)$, who applied to this class of rings his general structure theory [5]. Further results were obtained by I. Kaplansky $[9 ; 10]$ with the help of topological methods. He also deals, more generally, with $\pi$-regular $\left({ }^{2}\right)$ rings. In the present paper additional information concerning the structure of algebraic algebras is derived, and the theory is extended to the wider class of $I$-rings, that is, rings with nonzero idempotents in every non-nil right ideal.

Our starting point is the observation that if a semi-simple $I$-ring contains a nilpotent element $a$ of index $m$, then the ideal generated by $a$ contains a system of $\mathrm{m}^{2}$ matrix units. One of the consequences is then the following result: If $S$ is a semi-simple $I_{1}$-ring (i.e., an $I$-ring with bounded index), then $S$ is weakly reducible, that is, every nonzero ideal contains an ideal which is isomorphic with a total matrix ring of finite degree over an $I$-ring that does not contain nonzero nilpotent elements. The degrees of these "matrix ideals" are bounded, and their maximum is equal to the maximum of the indices of the nilpotent elements of $S$. By combining the theorem on matrix units with an argument due to Kaplansky (Lemma 3.2) that was used by him in his category theorems [10, Theorems 5.1 and 10.2] we show that also semi-simple $I_{2}$-rings (that is, $I$-rings with bounded index modulo each primitive ideal) are weakly reducible. In particular, for an $I_{3}$-ring (i.e., an $I$-ring with some polynomial identity modulo every primitive ideal) it follows that every nonzero ideal contains a matrix ideal satisfying a polynomial identity.

Further results are obtained for the so-called "faithful I-rings," that is, rings whose homomorphic images are $I$-rings. Generalizing a lemma due to Jacobson [6] concerning algebraic algebras, we show that any homomorphic image $S^{\prime}$ of a faithful $I$-ring $S$ with bounded index has also bounded index that does not exceed theindex of $S$. For faithful semi-simple $I_{1^{-}}, I_{2^{-}}$, and $I_{3}$-rings it is shown that there exists a finite or a transfinite ascending chain of ideals $\left\{A_{\sigma}\right\}$ with homogeneous $\rho$-regular difference rings $A_{\sigma+1}-A_{\sigma}$. Thus, as in the $\rho$-regular case that was considered by Kaplansky, a certain reduction is

Presented to the Society, December 29, 1950 under the title On rings with bounded index; received by the editors January 5, 1952 and, in revised form, June 19, 1952.

(1) Numbers in brackets refer to the bibliography at the end of the paper.

(2) A ring $S$ is $\pi$-regular if for any $a \in S$ there is some $b \in S$ and some integer $n$ (depending on $a$ ) such that $a^{n} b \cdot a^{n}=a^{n}$ (compare [14]). 
effected to strongly regular rings $\left({ }^{3}\right)$. Incidentally, a strongly regular ring can be characterized as a faithful $I$-ring without nonzero nilpotent elements. We note also that for faithful $I$-rings any $I_{1}$-ring is an $I_{2}$-ring whose primitive images have a common upper bound for their indices.

Apart from the greater generality $\left(^{4}\right)$ which is achieved by dealing with faithful $I$-rings rather than with $\pi$-regular rings, we have here also the advantage that the structural theorems concerning the ascending chains mentioned above have their converses. Thus, e.g., any semi-simple ring possessing an ascending chain $A_{\sigma}$ with homogeneous difference rings $A_{\sigma+1}-A_{\sigma}$ is a faithful $I_{2}$-ring.

The last two sections are devoted to the problem of the local finiteness of algebraic algebras. It is shown that any algebra $S$ possesses a maximal, locally finite ideal $K(S)$, called the locally finite kernel of $S$. This ideal contains also all one-sided locally finite ideals of $S$, and $K[S-K(S)]=0$. This notion, which seems to be of independent interest, combined with some of the structural results of the previous sections, leads to simplified, purely algebraic proofs of Kaplansky's theorems concerning the local finiteness of algebraic algebras.

\section{LIST OF NOTATIONS AND ABBREVIATIONS}

$I$-ring $=$ ring with nonzero idempotents in every non-nil right ideal.

Plain ring $=I$-ring without nonzero nilpotent elements.

Matrix ideal=ideal with unit, isomorphic with a total matrix ring of finite degree over a plain ring.

Index of $S=i(S)=$ maximum of the indices of the nilpotent elements in $S$. $I_{1}$-ring $=I$-ring with finite index.

$I_{2}$-ring $=I$-ring with finite index modulo every primitive ideal.

$I_{3}$-ring $=I$-ring with a polynomial identity modulo every primitive ideal.

WR-ring = weakly reducible ring (see Definition 3.1).

E-ring $=$ elementary ring (see Definition 4.1).

PE-ring $=$ pseudo elementary ring (see Definition 4.2).

$\mathrm{PE}$-chain = pseudo elementary chain (see Definition 4.3).

$F I$-ring $=$ faithful $I$-ring (i.e., any homomorphic image is an $I$-ring).

Strong PE-ring, strong PE-chain (see Definition 5.1).

FWR-ring = faithful weakly reducible ring (see Definition 5.2).

The locally finite kernel (see Definition 6.1).

2. Systems of matrix units. In the present section we consider a general $I$-ring, i.e., a ring with nonzero idempotents in every non-nil right ideal. An $I$-ring without nonzero nilpotent elements is called plain. The radical of

( $\left.{ }^{3}\right)$ A ring is strongly regular if for any $a$ there is some $b$ so that $a=a^{2} b$.

(4) I am indebted to the referee for the remark that by a method similar to that used by I. Kaplansky in his paper on Regular Banach algebras (Ind. Math. Soc. vol. 22 (1948) pp. 5762) it can be deduced that a Murray-von Neumann factor of type $\mathrm{II}_{1}$ is a non- $\pi$-regular $I$-ring. 
any ring has no nonzero idempotents [5]; hence the radical of an $I$-ring is a nil ideal, and any plain ring is semi-simple. One finds that in an $I$-ring also every left ideal $L$ contains a nonzero idempotent, i.e., the definition of an $I$-ring is symmetric. Indeed, if $a \in L, a$ non nilpotent, then the right ideal $a S$ contains an idempotent $e=a b \neq 0$. The element $e^{\prime}=b e a \neq 0$ is then an idempotent in $L$. It follows further that any nonzero right or left ideal in a semisimple $I$-ring contains a nonzero idempotent. One easily obtains:

Lemma 2.1. Let $S$ be an I-ring and $A$ either an ideal in $S$ or a subring of the form $e S e$,where $e$ is an idempotent. Then $A$ is also an I-ring. If $S$ is semi-simple, so is $A$.

\section{LEMMA 2.2. Every idempotent of a plain ring lies in the center $\left({ }^{5}\right)$.}

In the following we shall be concerned with systems of matrix units, i.e., systems of $n^{2}$ elements $e_{i k}$ which are subject to the following relations:

$$
e_{i j} e_{j^{\prime} k}=\left\{\begin{array}{lll}
0 & \text { if } & j \neq j^{\prime} \\
e_{i k} & \text { if } & j=j^{\prime}
\end{array}\right.
$$

THeOREM 2.1. Let $S$ be an I-ring and $N$ the radical of $S$. If $a$ is a nilpotent element of index $n$ (i.e., $a^{n}=0, a^{n-1} \neq 0$ ) and if $a^{n-1} \notin N$, then the ideal (a) generated by a contains a system of $n^{2}$ matrix units.

Proof. By $a^{n-1} \notin N$ we have also $a^{n-1} S \nsubseteq N$, i.e., $a^{n-1} S$ contains an idempotent $e_{11}=a^{n-1} b_{1} \neq 0, b_{1}=b_{1} e_{11}$. As is readily seen, the $n-1$ elements $d_{i}^{(1)}$ $=a^{n-i} b_{1} a^{i-1}, i=2, \cdots, n$, are also nonzero idempotents, and clearly $d_{i}^{(1)} e_{11}$ $=0, i=2, \cdots, n$. Suppose that for $n>k>1$ the element $b_{k}$ has been fixed such that $e_{11}=a^{n-1} b_{k}, b_{k} e_{11}=b_{k}$, and the $k-1$ idempotents $e_{i i}=a^{n-i} b_{k} a^{i-1}$, $i=1, \cdots, k-1$, are orthogonal. By putting $d_{j j}^{(k)}=a^{n-j} b_{k} a^{j-1}, j=k, \cdots, n$, we get

$$
\begin{aligned}
& e_{s s} e_{t t}=0, \quad \text { for } s \neq t \text { and } s, t \leqq k-1 \text {, } \\
& d_{j j}^{(k)} e_{i i}=0, \quad \text { for } k \leqq j \leqq n \text { and } i \leqq k-1 \text {, } \\
& d_{j j}^{(k)} d_{r r}^{(k)}=0, \text { for } r<j .
\end{aligned}
$$

By the first of these relations we obtain

$$
a^{n-s} b_{k} a^{n+s-t-1} b_{k} a^{t-1}=0
$$

for $s \neq t$ and $s, t \leqq k-1$.

Multiplication of (3) by $b_{k} a^{s-1}$ on the left and by $a^{n-t} b_{k}$ on the right yields $b_{k} a^{n+s-t-1} b_{k}=0$, or

$$
b_{k} a^{i} b_{k}=0 \quad \text { for } i \neq n-1 \text { and } i \geqq n-k+1 .
$$

Since $e_{i i} d_{k \mathbf{k t}}^{(\mathbf{k})}=a^{n-i} b_{k} a^{i-1} a^{n-k} b_{k} a^{k-1}=a^{n-i} b_{k} a^{n+i-k-1} b_{k} a^{k-1}$, it follows by (4) that

(5) This holds more generally for any ring without nonzero nilpotent elements (compare [4]). 


$$
e_{i i} d_{k k}^{(k)}=0
$$

for $2 \leqq i \leqq k-1$.

We put $e_{k k}=d_{k k}^{(k)}-e_{11} d_{k k}^{(k)}$ and $b_{k+1}=b_{k}-a^{k-1} b_{k} a^{n-k} b_{k}$, then we have $b_{k+1}=b_{k+1} e_{11}$ and by (2), (4), and (5) it follows readily that $e_{i i}=a^{n-i} b_{k+1} a^{i-1}, i=1, \cdots, k$, and that these $k$ nonzero idempotents are orthogonal. Consequently, together with the $n-k$ idempotents $d_{i i}^{(k+1)}=a^{n-i} b_{k+1} a^{i-1}, i=k+1, \cdots, n$, they are subject to relations (2), where $k$ is replaced by $k+1$. Thus we have shown by induction that there exists an element $b_{n}=b=b e_{11}$ such that in accordance with (2) and (4) we have

$$
\begin{aligned}
e_{s s} e_{t t}=0 & \text { for } s \neq t, \text { wherein } e_{i i}=a^{n-i} b a^{i-1}, i=1, \cdots, n, \\
b a^{i} b=0 & \text { for } i \geqq 0 \text { and } i \neq n-1 .
\end{aligned}
$$

With the aid of these relations one readily verifies that the $n^{2}$ elements $\boldsymbol{e}_{i k}=a^{n-i} b a^{k-1}, i, k=1, \cdots, n$, constitute a system of matrix units lying in the ideal (a).

Remark. Conversely, if an ideal contains the $n^{2}$ elements of a matrix system (1), then it also contains nilpotent elements of index $n$, e.g., the element $a=\sum_{i=1}^{n-1} e_{i} e_{i+1}$.

LeMмa 2.3. If a semi-simple I-ring $S$ contains a system of $n^{2}$ matrix units $e_{i k}, i, k=1, \cdots, n$, such that (a) either the ring $e_{11} S e_{11}$ is not plain, or (b) the ring $e_{11} S e_{11}$ is plain but the element $e=\sum_{i=1}^{n} e_{i i}$ is not in the center, then there exists a system of $(n+1)^{2}$ matrix units $d_{i k}, i, k=1, \cdots, n+1$, such that $d_{11} \in e_{11} S e_{11}$.

Proof. (a). In this case $e_{11} S e_{11}$ contains a nilpotent element of index $t>1$, and thus by Lemma 2.1 and Theorem 2.1 there exists in $e_{11} S e_{11}$ a system of $t^{2}$ matrix units $f_{i k}, i, k=1, \cdots, t$. Put $m=n t$, then the system of $m^{2}$ elements $d_{(i-1) t+j,(r-1) t+k}=e_{i 1} f_{j k} e_{1 r} ; i, r=1, \cdots, n ; j, k=1, \cdots, t$, satisfies (1), and the system $d_{i k}, i, k=1, \cdots, n+1$, is the required one.

(b). Consider the Peirce decomposition

$$
S=e S \oplus R=S e \oplus L, \quad e R=L e=0 .
$$

By (7) we have $S e=e S e \oplus R e$ and $e S=e S e \oplus e L$. Since $S e \neq e S$, either $R e \neq 0$ or $e L \neq 0$. We may assume that $\operatorname{Re} \neq 0$, i.e. $\left(^{6}\right), \operatorname{ReS} \neq 0$. Let $e^{*}$ denote a nonzero idempotent in $\operatorname{ReS}$, and put $f_{n+1}{ }_{n+1}=e^{*}-e^{*} e$; then by $e \cdot e^{*}=0$ and $e^{*} \operatorname{Re} S$ $\neq 0$ we have

$$
\begin{aligned}
f_{n+1 n+1}^{2}=f_{n+1 n+1} ; & e_{i i} f_{n+1 n+1}=f_{n+1 n+1} e_{i i}=0, \\
i & =1, \cdots, n, \text { and } f_{n+1 n+1} S \cdot e S \neq 0 .
\end{aligned}
$$

For some $j$ we have therefore $f_{n+1}{ }_{n+1} S \cdot e_{j j} S \neq 0$, or $0 \subset f_{n+1}{ }_{n+1} S e_{j 1} e_{11} e_{1 j} S$

(6) This is a consequence of semi-simplicity. 
$\subseteq f_{n+1}{ }_{n+1} S e_{11} S$, i.e., $f_{n+1}{ }_{n+1} S \cdot e_{11} \cdot S \neq 0$, which also implies( $\left(^{5}\right)$ that $e_{11}$ - $S f_{n+1}{ }_{n+1} S e_{11} \neq 0$. Consequently there exists a pair of elements $f_{1 n+1}$ and $f_{n+11}$ such that $f_{1{ }_{n+1}} \in e_{11} S f_{n+1}{ }_{n+1}, f_{n+1} \in f_{n+1}{ }_{n+1} S e_{11}$ and $f_{1 n+1} f_{n+11} \neq 0$. Since $e_{11} S e_{11}$ is a semi-simple $I$-ring, there exists a nonzero idempotent $d_{11}$, such that for a suitable $a_{11} \in e_{11} S e_{11}$ we have $d_{11}=f_{1 n+1} f_{n+1} a_{11}=d_{11} f_{1 n+1} f_{n+11}$ $a_{11} d_{11}$. Now put $d_{1 n+1}=d_{11} f_{1 n+1}, d_{n+11}=f_{n+1}{ }_{1} a_{11} d_{11}$; then

$$
d_{11} d_{1 n+1}=d_{1 n+1}, d_{n+11_{11}} d_{11}=d_{11}, \quad d_{1 n+1} d_{n+11}=d_{11} \in e_{11} S e_{11} .
$$

By Lemma 2.2 and (8) we also have $d_{11} e_{11} d_{11}=d_{11}$, or $d_{11}=d_{11} e_{1 i} e_{i 1} d_{11}$ so that by setting $d_{1 i}=d_{11} e_{1 i}, d_{i 1}=e_{i 1} d_{11}$ for $i=1, \cdots, n$ it follows in view of (8) that

$$
d_{1 i} d_{i 1}=d_{11}, \quad d_{11} d_{1 i}=d_{1 i}, \quad d_{i 1} d_{11}=d_{i 1}, \quad i=1, \cdots, n+1 .
$$

If we finally put $d_{i k}=d_{i 1} d_{1 k}, i, k=1, \cdots, n+1$, we obtain the required system of matrix units.

Definition 2.1. An ideal $A$ of a ring $S$ will be referred to as a matrix ideal of degree $n$ if $A$ has a unit and is isomorphic to a total matrix ring of degree $n$ over a plain $\operatorname{ring}\left({ }^{7}\right)$.

LemMA 2.4. If an idea $A$ of a ring $S$ contains a matrix system $e_{i k}, i, k$ $=1, \cdots, n$, such that the ring $e_{11} S e_{11}$ is plain and the idempotent $e=\sum_{i=1}^{n} e_{i i}$ is in the center of $S$, then $A$ contains a matrix ideal of $S$ of degree $n$.

Proof. For any element $t_{11}=e_{11} \cdot S \cdot e_{11}$ put $t=\sum_{i=1}^{n} e_{i 1} t_{11} e_{1 i}$; then the set of the $t$ 's constitutes a ring $T$ such that $T \cong e_{11} S e_{11}$, i.e., $T$ is plain. Clearly $e_{i k} t=t e_{i k}$ for $t \in T$ and the ideal $B$ generated by the $e_{i k}$ which coincides with the set of all elements of the form $\sum e_{i k} t_{i k}, t_{i k} \in T$, is then the required matrix ideal.

TheOREM 2.2. If $A$ is a nonzero ideal in a semi-simple I-ring $S$, then either $A$ contains a matrix ideal of $S$ or $A$ contains an infinite sequence of matrix unit systems $e_{i k}^{(j)}, i, k=1, \cdots, j ; j=1,2,3, \cdots$, such that $e_{11}^{(j+1)} \in e_{11}^{(j)} S e_{11}^{(j)}$.

Proof. Suppose that no matrix ideal lies in $A$. We begin the construction of the required infinite sequence by choosing an idempotent $e_{11}^{(1)} \neq 0$ in $A$. This is the first (one element) system of the sequence. Since by Lemma 2.4 either $e_{11}^{(1)} S e_{11}^{(1)}$ is not plain or $e_{11}^{(1)}$ is not in the center, it follows by Lemma 2.3 that there exists a matrix system $e_{i k}^{(2)}, i, k=1,2$, such that $e_{11}^{(2)} \in e_{11}^{(1)} S e_{11}^{(1)}$. Thus by induction we obtain the required sequence.

3. Weakly reducible rings. A ring $S$ is said to be of finite index $i(S)$ if the indices of the nilpotent elements of $S$ are bounded and their maximum is equal to $i(S)$. Otherwise $i(S)=\infty$. For $S=0$ we set $i(0)=0$. We shall refer to an $I$-ring of finite index as an $I_{1}$-ring. An $I$-ring $S$ such that for each primitive

(7) The following properties of a matrix ideal, common to all ideals with unit, will be often used in the sequel: I. If $T$ is an ideal in a ring $S$, and $A$ is an ideal with unit in $T$, then $A$ is an ideal in $S$. II. Any matrix ideal is a direct summand. 
ideal $P$ the index $i(S-P)$ is finite (depending on $P$ ) will be referred to as an $I_{2}$-ring. Finally, an $I_{3}$-ring is an $I$-ring such that for each primitive ideal $P$ the ring $S-P$ satisfies some polynomial identity (depending on $P$ ). In this case it follows by a theorem due to Kaplansky [7] that $S-P$ has a finite dimension $n^{2}$ over its center, so that $S-P$ is a total matrix ring of degree $m \leqq n$ over a division ring. Thus any $I_{3}$-ring is an $I_{2}$-ring. We also note (see [1]) that under this assumption $S-P$ satisfies the "standard identity" $\sum \pm x_{i_{1}} x_{i_{2}} \cdots x_{i_{2 n}}=0$, where the sign is positive for even permutations of the sequence $(1,2, \cdots, 2 n)$ and negative for odd permutations of this sequence. Finally we quote that the same identity holds for all primitive rings $S-P^{\prime}$ which have dimension $\leqq n^{2}$ over the respective center.

The principal aim of the present section is to show that the three classes of $I$-rings which we have just mentioned are instances of the class of the so-called weakly reducible rings, which are defined as follows:

Definition 3.1. Let $N^{\prime}$ denote the maximal nil-ideal of a ring $S$. We say that $S$ is weakly reducible (in short: $S$ is a WR-ring) if each nonzero ideal $A$ of $S-N^{\prime}$ contains a matrix ideal.

Any plain ring is evidently a semi-simple WR-ring. Also the following lemma is readily verified and its proof may be omitted.

LEMMa 3.1. Let $T$ be a ring without nilpotent elements other than zero and denote by $S=T_{n}$ the total matrix ring of degree $n$ over $T$. Then $S$ is a semi-simple $I$-ring if and only if $T$ is plain. Moreover, if $T$ is plain then $S$ is a semi-simple WR-ring. If $S$ is a primitive WR-ring, then $T$ is a division ring.

The following theorem is almost an immediate consequence of the definition.

THEOREM 3.1. If $S^{\prime}$ is weakly reducible and $N^{\prime}$ is the maximal nil-ideal of $S^{\prime}$, then the ring $S=S^{\prime}-N^{\prime}$ has the following properties:

(a) $S$ is a semi-simple I-ring (i.e., $N^{\prime}$ is the radical of $S^{\prime}$ ).

(b) $S$ is a subdirect sum of matrix rings of finite degree over plain rings.

(c) $S$ is primitive if and only if $S$ is isomorphic with a total matrix ring of finite degree over a division ring.

Proof. (a) Each nonzero ideal $A$ contains an idempotent $\neq 0$ (e.g., the unit of a matrix ideal which lies in $A$ ), hence $S$ is semi-simple. If $R$ is a nonzero right ideal in $S$, then $S R \neq 0\left(^{6}\right)$ and contains a matrix ideal $M \neq 0$. It follows that $R M \neq 0$. Since $M$ is a semi-simple $I$-ring (Lemma 3.1), the right ideal $R M$ of $M$ contains a nonzero idempotent, and this latter lies in $R$.

(b) Since any matrix ideal $M$ has a unit, we can write $S=M \oplus \tilde{M}$. Denote by $B$ the intersection of all the $\tilde{M}$ 's. If $B \neq 0$, then $B \supseteq M_{0}$, where $M_{0}$ is a matrix ideal. Write $S=M_{0} \oplus \tilde{M}_{0}$, then $\tilde{M}_{0} \supseteq B$. On the other hand by definition $B \subseteq \tilde{M}_{0}$. Hence we must have $B=0$, and this implies the statement under (b). 
(c) For any matrix ideal $M$ we have $S=M_{0} \oplus M$. If $S$ is primitive, we must have $S=M$ (compare [5, Lemma 4]). The statement follows now by Lemma 3.1.

We have to discuss now the problem which presents itself in connection with definitions 2.1 and 3.1 concerning the invariance of the degree of a matrix ideal. We need the following lemma:

Lemma 3.2. Let $T$ be a plain ring with unit e, and $S$ the $n$ by $n$ matrix ring over $T$. Then any one-sided inverse in $S$ is two-sided $\left({ }^{8}\right)$.

Proof. We have to show that if $a b=e$ in $S$, then $b a=e$. We use induction with respect to $n$. Our theorem is valid for $n=1$. Indeed, suppose that $b a \neq e$, then $a-b a^{2} \neq 0$, whereas $\left(a-b a^{2}\right)^{2}=0$, which is impossible, since $S(=T)$ is plain. Suppose now that $n>1$. If $c \in T, c \neq 0$, and $e^{\prime}$ is a nonzero idempotent in $c T$, then $e^{\prime}=e^{\prime} c c^{\prime \prime}, c^{\prime \prime} \in e^{\prime} T$ and (Lemma 2.2) $e^{\prime} T$ is a direct summand of $T$. By the foregoing remark we must have also $e^{\prime}=c^{\prime \prime} e^{\prime} c$, i.e., $e^{\prime} c$ is regular in $e^{\prime} T$. This shows that for some central nonzero idempotent $e^{\prime}$, all the elements of the matrices $a^{\prime}=e^{\prime} a, b^{\prime}=e^{\prime} b$, where $a^{\prime} b^{\prime}=e^{\prime}$, are either regular or zeros. To simplify notations write again $a, b, e$ instead of $a^{\prime}, b^{\prime}, e^{\prime}$ and put

$$
a=\left(\begin{array}{ll}
x & D \\
E & F
\end{array}\right), \quad b=\left(\begin{array}{ll}
y & G \\
H & K
\end{array}\right)
$$

where $F$ and $K$ are $n-1$ by $n-1$ matrices. We consider 3 cases:

Case I. $E=0$. Then by $a b=e$ we have $F K=I$, where $I$ is the $n-1$ by $n-1$ unity matrix with $e$ along the diagonal. By induction $F K=K F=I$. By $F H=0$ we have then $K F H=I H=H=0$. We have further $x y=e$, and hence (since $T$ is plain) also $y x=e$. Since finally $x G+D K=0$, it follows that $y(x G+D K) F=G F+y D=0$, which shows that $b a=e$.

Case II. $x \neq 0$. Since $x$ is regular, we get by putting

$$
c=\left(\begin{array}{cc}
e & 0 \\
-E x^{-1} & I
\end{array}\right) \text { and } a_{1}=c a, b_{1}=b c^{-1}
$$

the relation $a_{1} b_{1}=e$. We may assume (passage to a suitable direct summand!) that the elements of the matrices $a_{1}$ and $b_{1}$ are either zeros or regular, and since $a_{1}$ has the form treated in case I, we have $a_{1} b_{1}=e=b_{1} a_{1}=b a=a b$.

Case III. $x=0, E \neq 0$. Put

$$
c=\left(\begin{array}{ll}
e & M \\
0 & I
\end{array}\right)
$$

(8) The author is indebted to the referee for the present form of this lemma and the following Theorem 3.2. Originally these results were proved under the restrictive assumption that either $S$ or every primitive image of $S$ is of finite index. In these cases Lemma 3.2 is an easy consequence of a result due to N. Jacobson [7]. 
where $M$ is chosen so that $M E \neq 0$. By setting $a_{1}=c a$ and passing eventually to a suitable direct summand, we reduce this case to case II.

We have shown so $\operatorname{far}\left({ }^{9}\right)$ that for a suitable central nonzero idempotent $e_{1}$ the elements $a_{1}=a e_{1}, b_{1}=b e_{1}$ are subject to the relation $a_{1} b_{1}=b_{1} a_{1}=0$, or $(a b-b a) e_{1}=0$. Consider the ideal $A$ generated in $S$ by $a b-b a$. Since $S$ is a semi-simple WR-ring (Lemma 3.1 ) it follows that $A$ contains a matrix ideal $e_{2} S$, where $e_{2}$ is a nonzero central idempotent of $S$. For the elements $a_{2}=a e_{2}$, $b_{2}=b e_{2}$ we have $a_{2} b_{2}=e_{2}$. Hence by above considerations there exists in the matrix ring $e_{2} S$ over $e_{2} T$ a central nonzero idempotent $e_{3}$ so that by putting $a_{3}=a e_{3}, b_{3}=b e_{3}$ we get $a_{3} b_{3}=b_{3} a_{3}$, or $(a b-b a) e_{3}=0$. This implies that $A e_{3}=0$, which is a contradiction, since $e_{3} \in A$. Hence we must have $A=0$, or $a b-b a$ $=0$, q.e.d.

THEOREM 3.2. Suppose that a ring $S$ with a unit element has two isomorphic representations as a total matrix ring over plain rings, with degrees $n$ and $m$. Then $n=m$.

Proof. Our assumption implies that we may write $S=\sum_{i, k=1}^{n} e_{i k} T$ $=\sum_{i, k=1}^{m} d_{i k} U$, where $T$ and $U$ are plain rings, whose elements commute with the matrix units $e_{i k}$ and $d_{i k}$ respectively. We have to show that $m=n$. By $0 \neq e_{11} S S=e_{11} S \sum_{i=1}^{m} d_{i i} S$ it follows for some $r$ that $0 \neq e_{11} S d_{r r} S$ $=e_{11} S d_{r 1} d_{11} d_{1 r} S \subseteq e_{11} S d_{11} S$, or $e_{11} S d_{11} S \neq 0$. Similarly we get $0 \neq e_{11} S d_{11} S e_{11}$. For some $a_{11} \in e_{11} S d_{11}$ we have then $0 \subset a_{11} d_{11} S e_{11} \subseteq e_{11} T e_{11}$. Since $e_{11} T e_{11} \cong T$, the nonzero right ideal $a_{11} d_{11} S e_{11}$ of $e_{11} T e_{11}$ contains a nonzero idempotent $e_{1}=a_{11} b_{11}$, where $b_{11} \in d_{11} S e_{11}$. We put $a=\sum_{i=1}^{n} e_{i 1} e_{1} a_{11} d_{1 i}, b=\sum_{i=1}^{n} d_{i 1} b_{11} e_{1} e_{1 i}$, and $e=a b$. Since $e_{1}$ is in the center of $e_{11} T e_{11}$ (Lemma 2.2), it follows that $e$ is in the center of $S$, and thus the elements $a, b, e$ satisfy with respect to the matrix ring $e S$ over $e T$ the conditions of Lemma 3.2. Hence we have $b a=e$. On the other hand, assuming that $n<m$ it would follow by the definition of $a$ and $b$ that $d_{n+1} b a \neq 0$, while $b a d_{n+1} 1=0$, which is a contradiction, since $e$ is in the center. This shows that we must have $n \geqq m$. Similarly $m \geqq n$, hence $m=n$, q.e.d.

Theorem 3.3. A semi-simple $I_{1}$-ring is a WR-ring. The maximal degree of the matrix ideals contained in $S$ is equal to the index $i(S)$ of $S$.

Proof. This is a consequence of Theorems 2.1 and 2.2.

Combining this theorem with Theorem 3.1 we obtain the following:

CoRollary. An $I_{1}$-ring is primitive if and only if it is isomorphic with a total matrix ring of finite degree over a division ring.

The following lemma is extracted from an argument due to Kaplansky (see [10, Theorems 5.1 and 10.2]) and is reproduced here for the convenience of the reader.

(9) This result is already sufficient to prove Theorem 3.2 . 
LEMMA 3.3. If a ring $S$ contains an infinite sequence of nonzero idempotents $e_{i}, i=1,2, \cdots$, such that $e_{i+1} \in e_{i} S e_{i}$, then there exists a primitive ideal that does not contain any of the $e^{\prime}$.

Proof. Denote by $R_{i}$ the right ideal consisting of all elements of the form $x-e_{i} x$, and consider the right ideal $R=\sum_{i=1}^{\infty} R_{i}$. Suppose that for some positive integer $n$ we have

$$
e_{1}=\sum_{j=1}^{n} x_{j}-e_{j} x_{j}
$$

Since $e_{i} e_{i+k}=e_{i+k} e_{i}=e_{i+k}$, it follows by multiplying (9) with $e_{n}$ that $e_{n}=0$, a contradiction. Hence $e_{1} \in R$, i.e., $R \subset S$. Any right ideal $R^{\prime}$ such that $S$ $\supset R^{\prime} \supseteq R$ evidently does not contain any of the $e^{\prime}$. If $R^{\prime}$ is a maximal right ideal of this kind, then the quotient $P=R^{\prime}: S$ (see [5, p. 310]) is contained in $R^{\prime}$ and $P$ is the required primitive ideal.

\section{TheOREm 3.4. Any semi-simple $I_{2}$-ring $S$ is a WR-ring.}

Proof. Suppose that $S$ is not a WR-ring and let $A$ be a nonzero ideal that does not contain matrix ideals. By Theorem 2.2 we know that $A$ contains an infinite sequence of matrix units systems $e_{i k}^{(j)}, i, k=1, \cdots, j ; j=1,2, \cdots$, such that $e_{11}^{(j+1)} \in e_{11}^{(j)} S e_{11}^{(j)}$. By Lemma 3.3 there exists a primitive ideal $P$ that does not contain any of the idempotents $e_{11}^{(j)}, j=1,2, \cdots$, which in view of $e_{11}^{(j)}=e_{1 i}^{(j)} e_{i k}^{(j)} e_{k_{i}}^{(j)}$ implies that $e_{i k}^{(j)} \notin P, i, k=1, \cdots, j$; hence $S-P$ contains nilpotent elements of index $j$. As this is valid for any $j$, it follows that the index of $S-P$ is infinite, which is the required contradiction.

Theorem 3.5. A semi-simple $I_{3}$-ring $S$ is a WR-ring. Any nonzero ideal $A$ in $S$ contains matrix ideals that satisfy a polynomial identity.

Proof. Since any $I_{3}$-ring is an $I_{2}$-ring, we know (Theorem 3.4) that $A$ contains a matrix ideal $M_{1}$. As $M_{1}$ has a unit, $M_{1}$ is a homomorphic image of $S$, and thus any primitive image of $M_{1}$ is also a primitive image of $S$ and satisfies therefore for some integer $n$ the standard identity

$$
\sum \pm x_{i_{1}} x_{i_{2}} \cdots x_{i_{2 n}}=0 \text {. }
$$

Following [10, Theorem 10.3], denote by $B$ the crosscut of all primitive ideals $P$ of $M_{1}$ such that (10) holds in $M_{1}-P$; then (10) holds in $M_{1}-B$ also. Thus if $B=0$, the theorem is proved. If $B \neq 0$, consider a matrix ideal $M_{2}, M_{2} \subseteq B$; then $\left(^{7}\right) \quad M_{1}=M_{2} \oplus M^{\prime}$. Denote by $Q$ any primitive ideal of $M_{2}$. By $M_{1}$ $-\left(Q \oplus M^{\prime}\right) \cong M_{2}-Q$ it follows that $Q \oplus M^{\prime}$ is a primitive ideal in $M_{1}$, and that if (10) holds in $M_{2}-Q$, then it holds in $M_{1}-\left(Q+M^{\prime}\right)$ also, i.e., $B \subseteq Q$ $\oplus M^{\prime}$, contradicting $B \supseteq M_{2} \supset Q$. Hence (10) does not hold in $M_{2}-Q$. Thus, assuming that the theorem is false, and given an arbitrary ascending sequence of integers, say $1,2,3, \cdots$, we can deduce the existence of an 
infinite sequence of matrix ideals $\left\{M_{i}\right\}$ with units $e_{i}$ such that $e_{i} \in e_{i-1} S e_{i-1}$ and any primitive image of $M_{n}$ does not satisfy (10). By Lemma 3.3 there exists a primitive ideal $P$ that does not contain any of the $e$ 's. By assumption $S-P$ is simple, hence $S=P+M_{n}, n=1,2, \cdots$. By $S-P=\left(P+M_{n}\right)-P$ $\cong M_{n}-\left(M_{n} \cap P\right)$ we know $\left.{ }^{10}\right)$ that $M_{n} \cap P$ is a primitive ideal in $M_{n}$ and hence does not satisfy (10). It follows therefore that $S-P$ does not satisfy (10) for any $n$, which is the required contradiction.

4. Elementary and pseudo elementary rings. In case a ring $S$ contains matrix ideals, it is natural to consider the sum $A$ of all such ideals and to study the behaviour of $S-A$. In this and in the next section such considerations will play an important role.

If $A_{1}$ and $A_{2}$ are ideals with the units $e_{1}$ and $e_{2}$, then the $e^{\text {'s are central }}$ idempotents, and so are the three orthogonal idempotents $d_{1}=e_{1}-e_{1} e_{2}$, $d_{2}=e_{2}-e_{1} e_{2}$ and $d_{3}=e_{1} e_{2}$. The central idempotent $d=\sum_{i=1}^{3} d_{i}$ is then the unit of the ideal $A_{1}+A_{2}$. This leads readily to the following lemma:

LEMma 4.1. The sum of a finite number of ideals with units is also an ideal with a unit. If a finite system of elements lies in an ideal $A$ that is a sum of ideals with units, then there exists an ideal $B$ with a unit, $B \subseteq A$, that contains all the elements of the system.

Various special cases of the following lemma will be repeatedly applied.

Lemma 4.2. Let $A$ be an ideal in an arbitrary ring $S$ and suppose that $A$ is the sum of a finite or an infinite number of ideals with units. For a finite set of elements $x_{i} \in S, i=1, \cdots, m$, let $f_{j}(x)=f_{j}\left(x_{1}, \cdots, x_{m}\right), j=1, \cdots, n$, denote $a$ set of polynomials in the $x$ 's (say with integral coefficients). If

$$
f_{j}\left(x_{1}, \cdots, x_{m}\right) \equiv 0 \bmod A,
$$

$j=1, \cdots, n$,

then there exists a system of elements $y_{i}, i=1, \cdots, m$, such that

$$
f_{j}\left(y_{1}, \cdots, y_{m}\right)=0, \quad y_{i} \equiv x_{i} \bmod A, j=1, \cdots, n, i=1, \cdots, m .
$$

Proof. By Lemma 4.1 the finite set $f_{j}(x), j=1, \cdots, n$, lies in an ideal $B$ with a unit such that $B \subseteq A$. It follows that $S=B \oplus C$. Write $x_{i}=u_{i}+y_{i}$, $u_{i} \in B, y_{i} \in C$; then evidently $f_{j}(x)=f_{j}(u)+f_{j}(y), f_{j}(u) \in B, f_{j}(y) \in C$. Since $f_{j}(x) \in B$, this implies that $f_{j}(y)=0$, which in view of $y_{i}-x_{i} \in B \subseteq A$ completes the proof.

In the above proof the $u$ 's and the $y$ 's are components of the $x$ 's in the direct sum $S=B \oplus C$. Hence we have:

CoROLlary 1. If in (11) the $x$ 's are in the center of $S$, then we can choose the $y$ 's in (12) so that they also belong to the center of $S$.

A special case of (11) is the congruence $x^{n} \equiv 0 \bmod A$. Hence we have:

(10) This is a special case of [9, Theorem 3.1]. 
Corollary 2. If $a$ is nilpotent of index $n$ modulo $A$, then for some $b \equiv a \bmod A$ we have $b^{n}=0$. Thus if $S$ is of finite index $i(S)$, then also $S-A$ is of finite index and we have

$$
i(S) \geqq i(S-A) .
$$

By combining Corollaries 1 and 2 we obtain:

Corollary 3. If $z$ is a central element of $S$ and if $z$ is nilpotent of index $n$ modulo $A$, then $S$ contains a central element which is nilpotent of index $n$. have:

Since every central nilpotent element lies in the radical of the ring, we

Corollary 4. If $S$ is semi-simple, then each central element which is nilpotent modulo $A$ lies in $A$.

Finally we state the following special case of Lemma 4.1.

COROLLARY 5. If $e_{i k}, i, k=1, \cdots, n$, constitutes a system of $n^{2}$ matrix units modulo $A$, then there exists a system of $n^{2}$ matrix units $d_{i k}, i, k=1, \cdots, n$, in $S$ such that $d_{i k} \equiv e_{i k} \bmod A$.

Definition 4.1. An ideal $A$ in a ring $S$ (in particular: $A=S$ ) is called elementary (in short: E-ideal, E-ring) if $A$ is a sum of matrix ideals.

Lemmas 3.1 and 4.1 readily lead to the following:

LEMMA 4.3. Any E-ring is a semi-simple WR-ring.

We need the following generalization of the notion of an E-ring:

Definition 4.2. Let $A$ be a semi-simple ideal in a ring $S$ (in particular: $A=S$ ) and denote by $M$ the sum of all matrix ideals of $S$ contained in $A$. If $A-M$ is a nil-ring, then $A$ is called pseudo-elementary (in short: PE-ideal, PE-ring). If all matrix ideals in $A$ are of a fixed equal degree, we refer to $A$ as homogeneous( $\left.{ }^{11}\right)$.

Any E-ring is evidently a PE-ring, and if a PE-ring has a unit, it is an E-ring. Simple examples show $\left({ }^{12}\right)$ that there exist PE-rings which are not E-rings. We prove:

Lemma 4.4. Any PE-ring is a semi-simple WR-ring. If $Z$ is the center of $S$, then $Z S$ is the sum of all matrix ideals, i.e., $S-Z S$ is a nil-ring. If $S$ is of finite index, then so is $S-Z S$ and $i(S) \geqq i(S-Z S)$. If $S$ is a $P E$-ideal in a ring $T$, and $T$ is of finite index, then so is $S$, and we have $i(T) \geqq i(T-S)$.

Proof. Denote by $M$ the sum of all matrix ideals of $S$, and consider any

(11) In the case of FI-rings, in particular, of $\pi$-regular rings, this definition agrees with that of Kaplansky (compare Theorem 5.6(e) of the present paper).

(12) Compare [9, Theorem 4.3, remark 2]. 
ideal $A \neq 0$. By $(A+M)-M \cong A-(A \cap M)$ it follows that $A-(A \cap M)$ is a nil-ideal. Since $S$ is semi-simple, this implies that $A \cap M \neq 0$ and similarly $0 \subset(A \cap M)^{2} \subseteq A M \subseteq M$. Since $M$ is an E-ring, it follows by Lemma 4.3 that the nonzero ideal $A M$ of $M$ contains a matrix ideal. Hence $\left({ }^{7}\right)$ so does $A$, which shows that $S$ is a WR-ring. By Lemma 4.2 , Corollary 4 it follows further that all central elements of $S$ lie in $M$, hence $Z S \subseteq M$. On the other hand $M$ is generated by the units of its matrix ideals, hence $Z S=M$. The inequality $i(S) \geqq i(S-Z S)$ follows now as a consequence of Lemma 4.2, Corollary 2. Finally, in case $S$ is a PE-ideal in a ring $T$ of finite index, we have in view of the fact that $S-Z S$ is a nil-ideal in $T-Z S$ the inequality $i(T-Z S) \geqq i[(T-Z S)-(S-Z S)]=i(T-S)$. By Lemma 4.2, Corollary 2 we have $i(T) \geqq i(T-Z S)$, hence $i(T) \geqq i(T-S)$, q.e.d.

In case a PE-ring $S$ has no nilpotent elements, $S$ is plain (see Theorem 3.1 and Lemma 4.4$)$ and by $1 \geqq i(S) \geqq i(S-Z S)$ and the nillity of $S-Z S$ we have $S=Z S$. Hence:

\section{Corollary. A PE-ring without nilpotent elements is a plain E-ring.}

One readily verifies that an ideal in a total matrix ring of degree $n$ over a plain ring with unit is also a total matrix ring of degree $n$ over some plain ring. The sum of two matrix ideals of the same degree is also a matrix ideal. Since the degrees of matrix ideals are invariant (see Theorem 3.2), it follows that the crosscut of two matrix ideals of different degrees is equal to 0 . In view of Lemmas 4.1 and 4.4 this leads readily to:

Lemma 4.5. Let $S$ be a PE-ring and $Z$ the center of $S$. If the primitive images of $S$ have finite indices, then $S$ is an $I_{2}$-ring and $Z S$ splits into a direct sum of homogeneous E-rings. If $S$ itself is of finite index, then $S$ is an $I_{1}$-ring and $Z S$ splits into a finite direct sum of homogeneous E-rings. A homogeneous PE-ring with unit is a matrix ring over a plain ring.

Definition 4.3. An ideal $A$ of a ring $S$ is said to possess an E-chain, respectively a PE-chain of length $\tau$, if there exists an ascending chain of ideals $\left\{A_{\sigma}\right\}$ in $S$ which begins at $A_{0}=0$ and terminates at $A_{\tau}=A$ such that $A_{\sigma+1}-A_{\sigma}$ is an E-ring, respectively a PE-ring, and any $A_{\sigma}$ with a limit ordinal $\sigma$ is defined by $A_{\sigma}=\bigcup_{\rho<\sigma} A_{\rho}$, respectively by $\tilde{A}_{\sigma}$, where $\tilde{A}_{\sigma}-\bigcup_{\rho<\sigma} A_{\rho}$ is a nil-ring and $A-\tilde{A}_{\sigma}$ has no nonzero nil-ideals.

TheOREM 4.1. A ring $S$ with a PE-chain and without nonzero nil-ideals is a semi-simple WR-ring.

Proof. Let $\left\{A_{\sigma}\right\}$ be a PE-chain of $S$ of length $\tau$ and $A$ a nonzero ideal in $S$. Each $A_{\lambda}$ has a PE-chain of length $\lambda$. We use induction with respect to the length $\tau$. For $\tau=1$ the theorem is true by Lemma 4.4. If $\tau$ is a limit ordinal and $U=\bigcup_{\rho<\tau} A_{\rho}$, then $S-U$ and hence also $(A+U)-U$ are nil-ideals. Since $S$ has no nonzero nil-ideals, it follows by $(A+U)-U \cong A-(U \cap A)$ that 
$U \cap A \neq 0$ and $U A \neq 0$, so that for some $\lambda$ we have $A_{\lambda} A \neq 0$. By induction $(\lambda<\tau) A_{\lambda} A$ and hence $\left.{ }^{7}\right)$ also $A$ contain matrix ideals, hence $S$ is a semi-simple WR-ring. If finally $\tau=\lambda+1$, then assume first that $A_{\lambda} A \neq 0$. In this case it follows (induction!) that the ideal $A_{\lambda} A$ and hence $\left({ }^{7}\right)$ also $A$ contains a matrix ideal. Consider now the case where $A_{\lambda} A=0$. In this case $\left(A_{\lambda} \cap A\right)^{2}=0$, and hence by the semi-simplicity of $A_{\lambda}$ (induction!) we have $A_{\lambda} \cap A=0$, which implies

$$
\left(A_{\lambda}+A\right)-A_{\lambda} \cong A .
$$

Now $A_{\lambda+1}-A_{\lambda}$ is a PE-ring, i.e., by Lemma 4.4 a WR-ring. Hence by the above isomorphism it follows that $A$ contains a matrix ideal, which completes the proof of the theorem.

Since each E-ring is a PE-ring, we have:

Corollary. A ring $S$ with an E-chain is a semi-simple WR-ring.

TheOREM 4.2. If $S$ is a ring with a finite index $i(S)$, and $A$ an ideal with a PE-chain, then

$$
i(S-A) \leqq i(S) .
$$

Proof. Let $\left\{A_{\sigma}\right\}$ denote a PE-chain of $A$ of length $\tau$. We use induction with respect to the length. If $\tau=1$, the theorem is true by Lemma 4.4. If $\tau=\lambda+1$, then by induction $i(S) \geqq i\left(S-A_{\lambda}\right)$. Since further $A_{\lambda+1}-A_{\lambda}$ is a PE-ring, we have by Lemma 4.4 the inequality $i\left\{\left(S-A_{\lambda}\right)-\left(A_{\lambda+1}-A_{\lambda}\right)\right\} \leqq i\left(S-A_{\lambda}\right)$, which combined with $\left(S-A_{\lambda}\right)-\left(A_{\lambda+1}-A_{\lambda}\right) \cong S-A_{\lambda+1}=S-A$ yields (13). If finally $\tau$ is a limit ordinal and if for some $c \in S$ we have $c^{n} \in A, c^{n-1} \in A$, then in view of the nillity of $A-\mathrm{U}_{\sigma<\tau} A_{\sigma}$ (see definition 4.3) it follows that for some $\sigma<\tau$ and for some positive integer $m$ we have $c^{n m} \in A_{\sigma}, c^{n-1} \notin A_{\sigma}$, i.e., $c$ is nilpotent of index $\geqq n$ modulo $A_{\sigma}$. Since $\sigma<\tau$, we have by induction $i\left(S-A_{\sigma}\right) \leqq i(S)$, i.e., $n \leqq i(S)$. Hence the indices of the nilpotent elements of $S-A$ are bounded by $i(S)$, q.e.d.

5. Homomorphism faithful $I$-rings. In this section we consider faithful $I$-rings (in short: $F I$-rings), that is, rings which are carried into $I$-rings by every homomorphism. By the transitivity property of homomorphisms it follows that any homomorphic image of an $F I$-ring is also an $F I$-ring.

Lemma 5.1. Let $S$ be an FI-ring and $A \neq 0$ a semi-simple ideal of finite index $i(A)$. Denote by $A_{1}$ the sum of all matrix ideals of $S$ that lie in $A$, and by $B$ any ideal such that $A \supseteq B \supseteq A_{1}$, and $B-A$ is a nil ring. Then

$$
i(A)>i(A-B) \text {. }
$$

Proof. The ideal $A$ is an $I_{1}$-ring (see Lemma 2.1) and hence (see Theorem 3.3) it contains matrix ideals. Any matrix ideal of $A$ is a matrix ideal of $S\left({ }^{7}\right)$. Thus $A_{1} \neq 0$. Now $B$ is a PE-ideal and we have by Lemma 4.4 the inequality 


$$
i(A-B) \leqq i\left(A-A_{1}\right) \leqq i(A) .
$$

Since $A$ is an $F I$-ring and $S \sim S-A_{1}$, we know that $S-A_{1}$ is an $I$-ring and thus (see Lemma 2.1) the ideal $A-A_{1}$ of $S-A_{1}$ is also an $I$-ring. Now set $\imath(A)=n$ and suppose that in (14) we have $i(A-B)=i(A)=n$; then also $i\left(A-A_{1}\right)=n$ and by Theorem 2.1 there exists in $A-A_{1}$ a system of $n^{2}$ matrix units $e_{i k}, i, k=1, \cdots, n$. Hence by Lemma 4.2 Corollary 5 there exists in $A$ a system of $n^{2}$ matrix units $d_{i k}$ such that

$$
d_{i k} \equiv e_{i k} \bmod A_{1},
$$$$
i, k=1, \cdots, n .
$$

Since $i(A)=n$, it follows (see remark to Theorem 2.1) that no systems of $m^{2}$ matrix units with $m>n$ do exist in $A$, so that in view of Lemmas 2.3 and 2.4 the ideal $A^{*}$ generated by the $d$ 's is a matrix ideal which lies in $A$. Since $A_{1}$ is defined as the sum of all matrix ideals which lie in $A$, we have $A_{1} \supseteq A^{*}$, which contradicts (15). This shows that we must have $i\left(A-A_{1}\right)<n$, q.e.d.

THEOREM 5.1. If $S$ is an FI-ring and $A \neq 0$ a semi-simple ideal with finite index $i(A)$, then $A$ possesses a PE-chain $\left\{B_{i}\right\}$ of finite length such that $0=B_{0}$ $\subset B_{1} \subset \cdots \subset B_{r}=A$ and $i\left(A-B_{s}\right)>i\left(A-B_{s+1}\right), r \leqq i(A)$.

Proof. Denote by $A_{1}$ the sum of all matrix ideals of $S$ contained in $A$; then as in the proof of Lemma 5.1 we know that $S-A_{1}$ and $A-A_{1}$ are $I$ rings. Hence the radical of $A-A_{1}$ is a nil-ideal which evidently generates a nil-ideal in $S-A_{1}$. Thus if $N$ is the ideal in $S$ such that $N \supseteq A_{1}$ and $N-A_{1}$ is the radical of $S-A_{1}$, then $B_{1}=N \cap A$ is a PE-ideal $\left({ }^{13}\right)$ of $S$ contained in $A$, and $A-B_{1}$ is semi-simple. The ideal $B_{1}$ is then the first term (after $0=B_{0}$ ) of the required PE-chain. Since a homomorphic image of an $F I$-ring is also an $F I$-ring, we can repeat the procedure with the $I$-ring $S-B_{1}$ and the semisimple ideal $A-B_{1}$. Thus in case $A-B_{1} \neq 0$ we can get hold of an ideal $B_{2}$ such that $B_{1} \subset B_{2} \subseteq A, B_{2}-B_{1}$ a $\mathrm{PE}$-ring and $A-B_{2}$ semi-simple. Since by Lemma 5.1 we have $i(A)>i\left(A-B_{1}\right)>i\left(A-B_{2}\right)$, this process terminates after at most $i(A)$ steps, q.e.d.

In the following we shall refer to $F I$-rings with finite index as $F I_{1}$-rings, and to $F I$-rings with finite index modulo each primitive ideal as $F I_{2}$-rings. Similarly $\mathrm{FI}_{3}$-rings are defined (compare $\$ 3$ ).

Theorem 5.2. If $S$ is a semi-simple $F I_{1}$-ring, then $S$ has a PE-chain $\left\{A_{i}\right\}$ of finite length $r$ such that $i\left(S-A_{i}\right)>i\left(S-A_{i+1}\right), r \leqq i(S)$.

Proof. This is an immediate consequence of Theorem 5.1.

REMARK. By combining this theorem with Lemma 4.5 we can supplement the chain $\left\{A_{i}\right\}$ to a chain $\left\{A_{j}^{*}\right\}$ by pushing in additional ideals between $A_{i}$ and $A_{i+1}$ wherever necessary, so that the new chain becomes a PE-chain with homogeneous PE-rings $A_{i+1}^{*}-A_{i}^{*}$. We can determine the $A^{*}$ 's such that

(13) Compare definition 4.2. 
again $i\left(S-A_{i}^{*}\right)>i\left(S-A_{i+1}^{*}\right)$, i.e., as before the length of $\left\{A_{i}^{*}\right\}$ does not exceed $i(S)$.

THEOREM 5.3. If $S$ is a semi-simple $F I_{1}$-ring and if $A$ is an ideal such that $S-A$ is semi-simple, then $S$ possesses a PE-chain that runs through $A$.

Proof. By Theorem 5.1 we can construct a PE-chain for $A$. By assumption, $S-A$ is semi-simple and again an $F I$-ring. Now use Theorem 4.2 to derive the inequality $i(S-A) \leqq i(S)$, i.e., $S-A$ is of finite index. By Theorem 5.2 the PE-chain for $A$ can now be extended so as to yield a PE-chain for $S$.

THEOREM 5.4. Any homomorphic image $S^{\prime}$ of an $F I_{1}$-ring $S$ is also an $F I_{1}$-ring, and $i\left(S^{\prime}\right) \leqq i(S)\left({ }^{14}\right)$.

Proof. Consider the ideal $A$ which is the kernel of the homomorphism $S \sim S^{\prime}$. If $N$ is the radical of $S$, then $A \cap N$ is the radical of $A$, i.e., the ideal $A-(A \cap N)$ of the $F I_{1}$-ring $S-(A \cap N)$ is semi-simple and possesses by Theorem 5.1 a PE-chain. Hence by Theorem 4.3 we have

$$
i[S-(A \cap N)] \geqq i\{[S-(A \cap N)]-[A-(A \cap N)]\} .
$$

By the "second law of isomorphisms" this is the same as

$$
i[S-(A \cap N)] \geqq i(S-A) .
$$

Now $A \cap N$ is a nil-ideal and it is therefore clear that $i[S-(A \cap N)] \leqq i(S)$ and this relation in conjunction with (16) yields $i(S) \geqq i(S-A)=i\left(S^{\prime}\right)$, q.e.d.

THEOREM 5.5. If $S$ is a plain FI-ring, then

(a) Any homomorphic image of $S$ is a plain FI-ring. Any primitive image of $S$ is a division ring.

(b) The ring $S$ and all its ideals are E-rings.

(c) Any right or left ideal in $S$ is two-sided.

(d) Any right ideal in $S$ which is generated by a finite set of elements has a unit, i.e., $S$ is regular and biregular $\left({ }^{15}\right)$.

(e) The ring $S$ is strongly regular. Conversely, a strongly regular ring is a plain FI-ring.

Proof. (a) This is an immediate consequence of Theorem 5.4 and the corollary to Theorem 3.3. (b) Since for an ideal $A \neq 0$ of $S$ we have $i(A)=1$, it follows by Theorem 5.1 that $A$ must be a PE-ring, and hence (Corollary to Lemma 4.4) an E-ring. (c) Let $R \neq 0$ denote a right ideal in $S$, then $R$ contains nonzero idempotents all of which lie in the center of $S$ (Lemma 2.2). Consider the set $A$ of all $x$ such that $x \in R, S x \subseteq R$, then $A$ is a maximal

${ }^{(14)}$ This is a generalization of Jacobson's lemma on algebraic algebras (compare [6, Lemma 1]).

$\left.{ }^{15}\right)$ A ring is biregular if every principal ideal is generated by a central idempotent. 
(two-sided) ideal of $S$ that lies in $R$. By Theorem 5.4 we have $i(S-A)$ $\leqq i(S)=1$, that is, $S-A$ is a plain $F I$-ring, hence (by (b)) an E-ring. Suppose that $R \supset A$, then $R$ contains an element $e$ that maps into a nonzero idempotent modulo $A$. By Lemma 4.2, Corollary 5 there exists an idempotent $d$ in $S$ such that

$$
d \equiv e \bmod A
$$

Since $d$ is in the center of $S$ (Lemma 2.2) and by (17) we have $d \in R$, it follows that $d \in A$, which contradicts (17). This shows that we must have $R=A$, that is, $R$ is two-sided. Similarly for a left ideal. (d) For a finite set $a_{1}, a_{2}, \cdots, a_{n}$ in $S$ consider the right ideal $A=\left(a_{1}, \cdots, a_{n}\right)_{r}$ generated by the $a$ 's. By (c) this ideal is two-sided and by (b) it is an E-ring. Hence by Lemma 4.1 we know that $A$ lies in an ideal $A^{*}$ with unit where $A \subseteq A^{*}$. Hence we have $A=A^{*}$, i.e., $A$ has a unit. Since this unit is a central idempotent (Lemma 2.2), the proof of (d) is complete. (e) For $a \in S$ consider the right ideal $A=a^{2} S$. By (c) we know that $A$ is two-sided and by Theorem 5.4 that $S-A$ has no nilpotent elements. Since $a^{3} \in A$, it follows therefore that $a \in A$, i.e., $a=a^{2} b$ for some $b$, that is, $S$ is strongly regular. Conversely ( $\left.{ }^{16}\right)$, if $S$ is strongly regular and $R \neq 0$ is a right ideal, then for $0 \neq a \in R$ we have $a=a^{2} b$, or $a(a b-a b a b)=0$. Then either $a b=a b a b$ or else one of the two elements $a b-a b a b,(a b-a b a b) a$ is $\neq 0$ and nilpotent. Since $S$ has no nilpotent elements we have $a b=a b a b$, i.e., $e=a b$ is an idempotent, hence in the center, hence $a=a e=e a$, i.e., $R$ contains a nonzero idempotent, and consequently $S$ is a plain $I$-ring. That $S$ is an $F I$-ring is an immediate consequence of the definition of strong regularity.

LEMMA 5.2. If $T$ is a ring with unit and without nilpotent elements other than zero, then the total matrix ring $S=T_{n}$ of degree $n$ over $T$ is an FI-ring if and only if $T$ is strongly regular. If this condition holds, then $S$ is regular and biregular, and each primitive image of $S$ is a total matrix ring of degree $n$ over a division ring $\left({ }^{17}\right)$.

Proof. Write in standard notation $S=\sum_{i, k=1}^{n} e_{i k} T$, where the $e^{\prime}$ s are matrix units commuting with the elements of $T$. Suppose first that $S$ is an $F I$-ring and consider a right ideal $R^{\prime} \neq 0$ in $T$. Then $R=\sum_{i=1}^{n} e_{1 i} R^{\prime}$ is a non-nil right ideal in $S$ and contains a nonzero idempotent $e=\sum_{i=1}^{n} e_{1 i} i_{1 i}$. The element $t_{11}$ is then a nonzero idempotent in $R^{\prime}$, which shows that $T$ is a plain ring. As is readily seen, each homomorphism of $T$ can be extended to a homomorphism of $S$, and this implies that any homomorphic image of $T$ is an $I$-ring. Hence $T$ is a plain $F I$-ring and is therefore by Theorem 5.5 strongly regular. To prove the second part of the lemma, we assume that $T$ is strongly regular

${ }^{(16)}$ Compare [2, Theorem 3.2].

(17) This implies the invariance of the degree of a matrix ideal in an FI-ring in the sense of Theorem 3.2. 
and have to show that $S$ is biregular and regular. To this end choose an element $a=\sum_{i, k} e_{i k} t_{i k} \in S$. The ideal $A$ in $S$ generated by $a$ coincides with the ideal generated by the ideal $A^{\prime}$ of $T$ which has as generators the elements $t_{i, k}, i, k=1, \cdots, n$. Since $A^{\prime}$ has a unit (Theorem 5.5) it follows that also $A$ has a unit, i.e., $S$ is biregular. Consider next the right ideal $a S$. The right ideal in $T$ which is generated by the first row of the matrix $\left(t_{i k}\right)$ has a unit $d_{1}$ (Theorem 5.5) and the right ideal $a S$ contains an idempotent of the form $e_{1}=e_{11} d_{1}+\sum_{i=2}^{n} e_{i 1} t_{i 1}^{(1)}$. Write $a=e_{1} a+\left(a-e_{1} a\right)$. The matrix $a_{1}=a-e_{1} a$ has zeros in the first row. The elements in the second row generate an ideal in $T$ with a unit $d_{2}$. The right ideal $a_{1} S$ contains an idempotent of the form $e_{2}^{1}=e_{22} d_{2}+\sum_{i=3}^{n} e_{i 2} t_{i 2}^{(2)}$, and we have $e_{1} e_{2}^{1}=0$. Put $e_{2}=e_{2}^{1}-e_{2}^{1} e_{1}$; then $a=e_{1} a$ $+e_{2} a+a_{2}$, where $e_{1}, e_{2}$ are orthogonal and $e_{i} a=0, i=1,2$. Furthermore, $a_{2}$ has zeros in the first two rows. Thus, after $n$ steps, we get a representation of the form $a=\sum_{i=1}^{n} e_{i} a$, where $e=\sum_{i=1}^{n} e_{i}$ is a left unit for $a$, i.e., $a=e a, e=a b$, and $a=a b a$, that is, $S$ is regular. Finally note that each primitive image of $S$ is a matrix ring of degree $n$ over a primitive image of $T$, and the latter is by Theorem 5.5 a division ring. This concludes the proof of the lemma.

Lemma 5.3. A PE-ring $S$ is an FI-ring if and only if each matrix ideal of $S$ is a matrix ring of finite degree over a strongly regular ring. If this condition is satisfied, then $S$ is a $\pi$-regular $\mathrm{FI}_{2}$-ring.

Proof. If the condition of the lemma holds, then by Lemma 5.2 each matrix ideal of $S$ is regular and hence also the sum $M$ of all these ideals (since each has a unit) is regular. Since $S-M$ is a nil-ring, we have for any $a \in S$ an equation $a^{m}=b \in M$, which by the regularity of $M$ implies $a^{m} c a^{m}=a^{m}$ for some $c$, i.e., $S$ is $\pi$-regular and thus an $F I$-ring. If, further, $P$ is a primitive ideal, then $P \nsupseteq M$ (since $S-M$ is nil), hence for some matrix ideal $M_{0}$ it follows that $\left(M_{0}+P\right)-P$ is primitive [5, Theorem 22]. By $\left(M_{0}+P\right)-P$ $\cong M_{0}-\left(P \cap M_{0}\right)$ we conclude $\left.{ }^{7}\right)$ that $M_{0}-\left(P \cap M_{0}\right)$ is a primitive image of $M_{0}$ and is therefore (Lemma 5.2) a matrix ring of finite degree over a division ring. Since $M_{0}-\left(P \cap M_{0}\right)$ has a unit, also $\left(M_{0}+P\right)-P$ has a unit and hence [5, Lemma 4] we must have $M_{0}+P=S$. This shows that $S-P$ is of finite index, i.e., $S$ is an $F I_{2}$-ring. Conversely, suppose that $S$ is an $F I$-ring; then as each matrix ideal $M_{0}$ of $S$ has a unit, $M_{0}$ is a homomorphic image of $S$ and is therefore also an $F I$-ring and hence (Lemma 5.2) a matrix ring of finite degree over a strongly regular ring. This completes the proof of the lemma.

Definition 5.1. A PE-ring which is also an $F I$-ring will be referred to as a strong $P E$-ring. A PE-chain $\left\{A_{\sigma}\right\}$ where all difference rings $A_{\sigma+1}-A_{\sigma}$ are strong PE-rings will be called a strong $P E$-chain.

THEOREM 5.6(18). Let $S$ be a semi-simple $F I_{1}$-ring with index $n=i(S)$, and

(18) Parts (b), (d) and (e) of this theorem generalize Kaplansky's results on $\pi$-regular rings (compare [9, Theorems $2.3,4.2$, and 4.3$]$ ). 
denote by $\left\{A_{i}\right\}$ a PE-chain (Theorem 5.2) of length $r$ such that $i\left(S-A_{i}\right)$ $>i\left(S-A_{i+1}\right)$. Then:

(a) Each matrix ideal in any of the rings $A_{i+1}-A_{i}$ is a homomorphic image of $S$. The chain $\left\{A_{i}\right\}$ is a strong PE-chain.

(b) Any primitive image of $S$ is a total matrix ring of degree $\leqq n$ over a division ring. Hence any $F I_{1}$-ring is a special $F I_{2}$-ring.

(c) The maximum of the degrees of the primitive images of $S$ is equal to $n$.

(d) If the primitive images of $S$ have equal degrees, then $r=1$ and $S$ is a strong homogeneous PE-ring.

(e) If $S$ has a unit and the primitive images of $S$ have equal degrees, then $S$ is a total matrix ring of degree $n$ over a strongly regular ring.

Proof. (a) The ring $S-A_{i}$ is a homomorphic image of $S$ and is therefore an $F I$-ring. If $M_{0}$ is a matrix ideal of $S-A_{i}$ that lies in $A_{i+1}-A_{i}$, then since $M_{0}$ has a unit, it is a direct summand of $S-A_{i}$ and is therefore a homomorphic image of $S-A_{i}$. Hence $M_{0}$ is an $F I$-ring and thus (Lemma 5.2) a matrix ring over a strongly regular ring. (b) If $S^{\prime}$ is a primitive image of $S$, then (Theorem 5.4) $i\left(S^{\prime}\right) \leqq i(S)$, which in view of the corollary to Theorem 3.3 implies that $S^{\prime}$ is a total matrix ring of degree at most equal to $n$ over a division ring. (c) By Theorem 3.2 we know that $S$ contains a matrix ideal $M_{0}$ of degree $n$. Any primitive image of $M_{0}$ is a primitive image of $S$ (compare the proof of (a)). Hence it follows (Lemma 5.2) that $S$ has primitive images of degree $n$. (d) This is a consequence of (a) and (c). (e) This is a consequence of (d) and Lemma 4.5 .

By the above theorem one is led to single out rings that possess a finite, or a transfinite, strong PE-chain. Our aim is to derive a necessary and sufficient condition for a ring to possess such a chain. We need the following:

Lemma 5.4. If for some ideal $A$ of a ring $S$ both $A$ and $S-A$ are I-rings, then also $S$ is an I-ring. If a ring $S$ is the union of an ascending chain of ideals $\left\{A_{i}\right\}$ each of which is an I-ring, then also $S$ is an I-ring.

Proof. Let $R$ be a non-nil right ideal in $S$. If the right ideal $R \cap A$ of $A$ is not nil, then $R \cap A$ and hence also $R$ possesses a nonzero idempotent. If $R \cap A$ is nil, then $R-(R \cap A)$ is not nil and in view of $(R+A)-A \cong R$ $-(R \cap A)$ the right ideal $(R+A)-A$ of $S-A$ contains a nonzero idempotent. Hence $R-(R \cap A)$ contains a nonzero idempotent, which implies (compare $[11$, Hilfsatz 3]) that also $R$ contains a nonzero idempotent. As to the second part of the lemma, note that if $R$ is a non-nil right ideal in $S$ then the nonnil right ideal generated by some non-nilpotent element of $R$ lies in some ideal $A_{i}$ and hence contains a nonzero idempotent that lies in $R$.

TheOREM 5.7. A semi-simple ring $S$ possesses a strong $P E$-chain if and only if $S$ is an $\mathrm{FI}_{2}$-ring.

Proof. Sufficiency: If $S$ is an $F I_{2}$-ring, then (Theorem 3.4) $S$ contains 
matrix ideals. Each of these matrix ideals is an $F I$-ring (compare the proof of Theorem 5.6(a)) and is therefore by Lemma 5.2 isomorphic with a total matrix ring over a strongly regular ring. Denote by $M$ the sum of all matrix ideals of $S$. As in the proof of Theorem 5.1 we determine the ideal $A_{1}$ such that $A_{1} \supseteq M, A_{1}-M$ is the radical of $S-M$, and $S-A_{1}$ is semi-simple. By Lemma 5.3 we know that $A_{1}$ is a strong PE-ring, and since along with $S$ also $S-A_{1}$ is a semi-simple $F I_{2}$-ring, we can continue the process and thus by transfinite induction $\left({ }^{19}\right)$ get a strong PE-chain for $S$.

Necessity: Consider a semi-simple ring $S$ with a strong PE-chain $\left\{A_{\sigma}\right\}$. By Theorem 4.1 we know that $S$ is an $I$-ring. We show next that $S$ is an $F I$-ring. To this end we have to prove that for any ideal $A$ the ring $S-A$ is an $I$-ring. We use induction with respect to the length $\tau$ of the chain $\left\{A_{\sigma}\right\}$. For $\tau=1$ we have $S=A_{1}$; hence $S$ is a strong PE-ring, hence an $F I$-ring, and thus $S-A$ is an $I$-ring. For $\tau=\lambda+1$ we have

$$
\left(A+A_{\lambda}\right)-A \cong A_{\lambda}-\left(A_{\lambda} \cap A\right) \text {. }
$$

By induction $A_{\lambda}$ is an $F I$-ring and hence the rings on both sides of (18) are $I$-rings. Now $S-A_{\lambda}$ is a strong PE-ring, i.e., an $F I$-ring, and hence the homomorphic image $\left(S-A_{\lambda}\right)-\left[\left(A+A_{\lambda}\right)-A_{\lambda}\right]$ of $S-A_{\lambda}$ is an $I$-ring. Since we have

$$
\left(S-A_{\lambda}\right)-\left[\left(A+A_{\lambda}\right)-A_{\lambda}\right] \cong S-\left(A+A_{\lambda}\right),
$$

it follows that $S-\left(A+A_{\lambda}\right)$ is an $I$-ring, and as we know already that also $\left(A+A_{\lambda}\right)-A$ is an $I$-ring, we conclude by Lemma 5.4 that $S-A$ is an $I$-ring. If $\tau$ is a limit ordinal, $\tilde{A}_{\tau}=\mathrm{U}_{\lambda<\tau} A_{\lambda}$, and $\tilde{A}_{\tau}^{\prime}=\mathrm{U}_{\lambda<\tau}\left(A_{\lambda}+A\right)$, then $S-\tilde{A}_{\tau}$ and hence also $S-\tilde{A}_{\tau}^{\prime}$ are both nil, i.e., $I$-rings. Now $\tilde{A}_{\tau}^{\prime}-A=\mathrm{U}_{\lambda<\tau}\left(A_{\lambda}+A\right)-A$, where each term is by induction (comprare (18)) an $I$-ring. Hence by Lemma 5.4 $\tilde{A}_{\tau}^{\prime}-A$ is an $I$-ring. In view of the nillity of $(S-A)-\left(\tilde{A}_{\tau}^{\prime}-A\right)$ this implies (Lemma 5.4) that also $S-A$ is an $I$-ring. Thus we have shown that $S$ is an $F I$-ring. We prove next that $S$ is an $I_{2}$-ring, i.e., that each primitive image of $S$ is of finite index. To this end suppose that $A$ is a primitive ideal and use again induction as before. For $\tau=1$ this is true by Lemma 5.3. For $\tau=\lambda+1$ assume first that $A \supseteq A_{\lambda}$. In this case $S-A$ is a primitive image of $S-A_{\lambda}$ and since this latter ring is by assumption a strong PE-ring, it follows by Lemma 5.3 that $S-A$ is of finite index. In case $A \supseteq A_{\lambda}$ we know that $\left(A+A_{\lambda}\right)$ $-A$ is a primitive ring (see [5, Theorem 22]) and by 18 it follows (induction!) in view of Lemma 5.3 that $\left(A+A_{\lambda}\right)-A$ is a total matrix ring of finite degree over a division ring, which implies that $A+A_{\lambda}=S$ and that $S-A$ is of finite index. Finally consider the case where $\tau$ is a limit ordinal; then for some

(19) Note that for a limit ordinal $\sigma$ we first determine $\tilde{A}_{\sigma}=\bigcup_{\rho<0} A_{\rho}$. Since $S-\tilde{A}_{\sigma}$ is an $I$-ring, the radical is nil, and we may fix $A_{\sigma}$ by assuming that $A_{\sigma} \supseteq \tilde{A}_{\sigma}$ and that $A_{\sigma}-\tilde{A}_{\sigma}$ should be the radical of $S-\widetilde{A}_{\sigma}$. Thus $S-A_{\sigma}$ becomes again a semi-simple $I_{2}$-ring, and we may proceed with the construction of the PE-chain. 
$\lambda<\tau$ we must have $A \nsupseteq A_{\lambda}$ since otherwise by $A \supseteq \tilde{A}_{\tau}=\mathrm{U}_{\lambda<\tau} A_{\lambda}$ it would follow that $S-A$ is nil, while $S-A$ is primitive. Thus with the help of (18) we conclude as before that $S-A$ is of finite index. This completes the proof of the theorem.

Remark. In the special case where the strong PE-chain $A_{i}$ is finite and each ring $A_{i+1}-A_{i}$ is of finite index it follows that $S$ is a semi-simple $F I_{1}$-ring, and we have obtained the converse of Theorem 5.2.

We have seen (Theorem 3.4) that for general semi-simple $I$-rings the condition that all primitive images are of finite index implies weak reducibility. Since this condition is inherited by each homomorphic image we have:

THEOREM 5.8. A semi-simple $\mathrm{FI}_{2}$-ring and all its homomorphic images are WR-rings.

In connection with the converse of this theorem one is led to single out the following class of rings:

Definition 5.2. A ring $S$ is called a faithful weakly reducible ring (in short: FWR-ring) if any homomorphic image of $S$ is weakly reducible.

LEMma 5.5. A ring $S$ without nonzero nilpotent elements is a FWR-ring if and only if $S$ is strongly regular. If $T$ is a ring with unit and without nilpotent elements, then the total matrix ring $T_{n}$ of degree $n$ over $T$ is a FWR-ring if and only if $T$ is strongly regular.

Proof. The proof is similar to that of Theorem 5.5 and Lemma 5.2. We omit the details.

We prove now the converse of Theorem 5.8.

THEOREM 5.9. Any FWR-ring $S$ is an $F I_{2}$-ring.

Proof. Denote by $N$ the maximal nil ideal of $S$. By definition the ring $S^{*}=S-N$ contains matrix ideals. Any matrix ideal $M_{0}$ is a semi-simple $I$-ring (Lemma 3.1). Since $M_{0}$ has a unit, $M_{0}$ is a homomorphic image of $S$ and is therefore a FWR-ring. Hence (Lemma 5.5) $M_{0}$ is a total matrix ring over a strongly regular ring. The sum $M$ of all matrix ideals of $S$ is therefore a regular ring (Lemmas 5.2 and 5.3). Now define the ideal $A_{1}$ by the requirements: $A_{1} \supseteq M, A_{1}-M$ is the maximal nil-ideal of $S-M$. Then $A_{1}-M$ is a strong PE-ideal and the WR-ring $S-A_{1}$ has no nonzero nil ideals. This process may be continued $\left({ }^{20}\right)$ and leads by transfinite induction to a strong PE-chain for $S$. In view of Theorem 5.7 this implies that $S^{*}$, and hence also $S$, is an $F I_{2}$-ring.

For the special case of a regular ring we have:

THEOREM 5.10. If the primitive images of a regular ring $S$ are of finite index, then $S$ possesses a strong $P E$-chain $\left\{A_{\sigma}\right\}$ such that $A_{\sigma+1}-A_{\sigma}$ is an E-ring

${ }^{(20)}$ Compare footnote 19 
for each $\sigma$, and for a limit ordinal $\sigma$ we have $A_{\sigma}=\bigcup_{\lambda<\sigma} A_{\lambda}$. Conversely, any ring that possesses a chain of this kind is a regular ring whose primitive images are of finite index.

Proof. A regular ring whose primitive images are of finite index possesses a strong PE-chain $\left\{A_{\sigma}\right\}$. This follows as a special case of Theorem 5.7. Now, any homomorphic image $S^{\prime}$ of a regular ring $S$ is also a regular ring, so that $S^{\prime}$ has no nonzero nil ideals. This implies that the PE-ring $A_{\sigma+1}-A_{\sigma}$ is actually an E-ring for any $\sigma$. Similarly it follows that for a limit ordinal $\sigma$ we have $A_{\sigma}=\mathrm{U}_{\lambda<{ }_{\sigma}} A_{\lambda}$ (compare definition 4.3). This shows that our strong PE-chain $\left\{A_{\sigma}\right\}$ is actually a strong E-chain. Conversely, suppose that $S$ is a ring that possesses a chain of the kind described in the theorem. Then $A_{\sigma+1}-A_{\sigma}$ is a sum of matrix ideals each of which is a regular ring (Lemma 5.2), which implies that also $A_{\sigma+1}-A_{\sigma}$ is regular. The regularity of $S$ follows now as a consequence of the following two facts that can be readily verified: (1) If $A$ is an ideal in a ring $T$ such that $A$ and $T-A$ are both regular, then also $T$ is regular $\left.{ }^{21}\right)$. (2) If a ring $T$ is the union of an ascending chain of ideals each of which is regular, then $T$ is also regular.

Corollary. All homomorphic images of an $\mathrm{FI}_{2}$-ring $S$ are semi-simple if and only if $S$ is regular.

Theorem 5.11. A semi-simple $F I_{3}$-ring possesses a strong $P E$-chain $\left\{A_{\sigma}\right\}$ such that $A_{\sigma+1}-A_{\sigma}$ satisfies a polynomial identity. Conversely, any semi-simple ring possessing a chain of this kind is an $\mathrm{FI}_{3}$-ring.

Proof. The proof is based on Theorem 3.5 and is similar to that of Theorem 5.7.

6. Locally finite ideals. Let $A$ be an algebra over a field $\Phi$. An element $a$ of $A$ is called algebraic if for some $\Phi$-polynomial $\left({ }^{22}\right) f(x)$ of degree $\geqq 1$ we have $f(a)=0$. An ideal $B$ of $A$ is called algebraic if all elements of $B$ are algebraic. Suppose that $B$ and $C$ are algebraic ideals in $A$. If $b \in B$ and $c \in C$, we have apparently for each positive integer $k$ the relation $(b+c)^{k}=b^{k}+c_{k}$, $c_{k} \in C$. If now $f(x)=\sum_{i=1}^{m} \alpha_{i} x^{i}, \alpha_{i} \in \Phi$, and $f(b)=0$, then $f(b+c)=f(b)$ $+\sum_{i=1}^{m} \alpha_{i} c_{i}=\sum \alpha_{i} c_{i}=c^{\prime}$, where $c^{\prime} \in C$. For a nonzero $\Phi$-polynomial $g(x)$ we have $g\left(c^{\prime}\right)=0$, i.e., $g\{f(b+c)\}=0$, which shows that $b+c$ is algebraic. The ideal $B+C$ is therefore algebraic. It follows now readily that the sum of all algebraic ideals is also an algebraic ideal. This maximal algebraic ideal of an algebra $A$ may be called the algebraic kernel of $A$ and denoted by $K^{\prime}=K^{\prime}(A)$. If for some $a^{\prime} \in A$ and for a nonzero $\Phi$-polynomial $f(x)$ we have $f\left(a^{\prime}\right)=a^{\prime \prime}$ $\in K^{\prime}$, then in view of the algebraicity of $K^{\prime}$ there exists a nonzero $\Phi$-polynomial $g(x)$ such that $g\left(a^{\prime \prime}\right)=0$. Hence $g\left\{f\left(a^{\prime}\right)\right\}=0$, i.e., $a^{\prime}$ is algebraic. This implies that $A-K^{\prime}$ has no algebraic ideals other than zero, and thus

(21) Compare [9, Theorem 4.4].

(22) That is, with coefficients in $\Phi$. 
we have proved:

Theorem 6.1. Any algebra $A$ contains a maximal algebraic ideal, the algebraic kernel $K^{\prime}(A)$ of $A$. The algebraic kernel of $A-K^{\prime}(A)$ is equal to 0 .

Any nilpotent element of an algebra is algebraic. Hence we have:

COROLlary. The maximal nil ideal of an algebra is a subset of the alegbraic kernel.

REMARK. It is not known whether or not the algebraic kernel contains also all one-sided algebraic ideals of an algebra.

If $M$ is a $\Phi$-module that lies in an algebra $A$, over $\Phi$, then the algebra $\{M\}_{\Phi}$ generated over $\Phi$ by the elements of $M$ coincides with the sum of all powers of $M$, or in short: $\{M\}_{\Phi}=\sum_{i=1}^{\infty} M^{i}$. The algebra $\{M\}_{\Phi}$ is apparently finite if and only if the module $M$ is finite and for some positive integer $r$ the relation $\{M\}_{\Phi}=\sum_{i=1}^{r} M^{i}$ holds. This latter relation is clearly equivalent with

$$
M^{i} \subseteq \sum_{i=1}^{r} M^{i}, \quad j=1,2, \cdots
$$

Another equivalent condition for the finiteness of $\{M\}_{\Phi}$ is that all powers of $M$ should lie in a fixed finite $\Phi$-module.

A subalgebra $B$ of $A$ is called locally finite (see [6]) if any finite $\Phi$-module of $B$ generates a finite (i.e., finite-dimensional) algebra. A locally finite algebra is evidently algebraic. It is not known whether the converse is true (see [6]).

LEMMA 6.1. If $R_{1}$ and $R_{2}$ are locally finite right ideals of an algebra $A$, then the sum $R_{1}+R_{2}$ is also a locally finite right ideal.

Proof. We have to show that if $M=\left[a_{1}, a_{2}, \cdots, a_{k}\right]$ is a finite $\Phi$-module that lies in $R_{1}+R_{2}$, then the algebra $\{M\}_{\Phi}$ is finite. To this end put $a_{i}=b_{i}+c_{i}$, where $b_{i} \in R_{1}, c_{i} \in R_{2}$ and consider the modules $M_{1}=\left[b_{1}, \cdots, b_{k}\right]$ and $M_{2}$ $=\left[c_{1}, \cdots, c_{k}\right]$. Evidently $M \subseteq M_{1}+M_{2}$. Consider the algebras $\left\{M_{1}\right\}_{\Phi}=Q_{1}$ and $\left\{M_{2}\right\}_{\Phi}=Q_{2}$. Since $R_{1}$ and $R_{2}$ are locally finite, it follows that $Q_{1}$ and $Q_{2}$ are finite, and hence that also the modules $P=Q_{1}+Q_{2}, P_{1}=Q_{1} Q_{2}$, and $P_{2}$ $=Q_{2} Q_{1}$ are finite. Since $P_{1} \subseteq R_{1}$ and $P_{2} \subseteq R_{2}$, it follows by the local finiteness of $R_{1}$ and $R_{2}$ that for some integer $n$ we have $\left\{P_{i}\right\}_{\Phi}=\sum_{j=1}^{n} P_{\uparrow}^{j} i=1,2$. Since, further, $Q_{1}$ and $Q_{2}$ are algebras, we have $Q_{1}^{j} \subseteq Q_{1}$ and $Q_{2}^{j} \subseteq Q_{2}$ for every $j$. Hence we have

$$
P^{m} \subseteq \sum_{i=1}^{n}\left(P_{1}^{i}+P_{2}^{i}\right)+\sum_{i=0}^{n}\left(P_{1}^{i} Q_{1}+P_{2}^{i} Q_{2}\right) ; m=1,2, \cdots ; P_{1}^{0}=P_{2}^{0}=\Phi .
$$

As the module on the right of (20) is finite, it follows that the algebra $\{P\}_{\Phi}$ is finite, and since $M \subseteq P$, we conclude that also the algebra $\{M\}_{\Phi}$ is 
finite. This completes the proof of the lemma.

Lemma 6.2. The sum of all locally finite right ideals of an algebra $A$ is a locally finite right ideal.

Proof. It follows by induction that the sum of any finite number of locally finite right ideals is also locally finite. If $M$ is a finite $\phi$-module that lies in the sum of all locally finite right ideals, then $M$ is contained in the sum of a finite number of these ideals. Hence $\{M\}_{\Phi}$ is finite, q.e.d.

Definition 6.1. The sum $K(A)$ of all locally finite right ideals of an algebra $A$ is called the locally finite kernel of $A$.

Lemma 6.3. If $R$ is a locally finite right ideal of an algebra $A$, then the ideal $A R+R$ is also locally finite.

Proof. By Lemma 6.1 it is sufficient to show that $A R$ is locally finite. Now consider a finite $\Phi$-module $Q=\left[c_{1}, \cdots, c_{k}\right]$ in $A R$. Then $c_{i}=\sum_{j=1}^{m_{i}} a_{i j} b_{i j}$, $a_{i j} \in A, b_{i j} \in R$. Put $Q_{1}=\left[\cdots, a_{i j}, \cdots\right], Q_{2}=\left[\cdots, b_{i j}, \cdots\right], L=Q_{1} Q_{2}$, and $M=Q_{2} Q_{1}$. Since $R$ is locally finite, $M$ is a finite module and $M \subseteq R$, it follows that for some integer $r$ relations (19) must hold. Hence we have

$$
L^{n} \subseteq \sum_{i=0}^{r} Q_{1} M^{i} Q_{2}, \quad n=1,2, \cdots ; M^{0}=\Phi .
$$

By (21) it follows that the algebra $\{L\}_{\Phi}$ is finite, and since we have $Q \subseteq L$, we conclude that $\{Q\}_{\Phi}$ is finite also, q.e.d.

THEOREM 6.2. The locally finite kernel is the maximal locally finite ideal of the algebra. This ideal contains all locally finite one-sided ideals of the algebra.

Proof. The proof follows immediately from Lemmas 6.2 and 6.3 in view of the fact that analogous lemmas hold for left ideals also.

Lemma 6.4. Let $D$ be a locally finite ideal in an algebra $A$. If $B$ is a subalgebra of $A$ such that $B \supseteq D$, and if the difference algebra $B-D$ is locally finite, then also $B$ is locally finite $\left.{ }^{23}\right)$.

Proof. Let $M$ be a finite $\Phi$-module, $M \subseteq B$. Since $B-D$ is locally finite, we have in view of (19) for some integer $r$ the relation $M^{r+1} \subseteq \sum_{i=1}^{r} M^{i}+D$. Hence if $c_{1}, c_{2}, \cdots, c_{m}$ is a $\Phi$-basis of the module $M^{r+1}$, we may put $c_{j}=c_{j}^{\prime}$ $+d_{j}$, where $c_{j}^{\prime} \in \sum_{i=1}^{r} M^{i}, d_{j} \in D$. Thus by setting $L=\left[\cdots, d_{j}, \cdots\right]$ we get

$$
M^{r+1} \subseteq \sum_{i=1}^{r} M^{i}+L
$$

Since $D$ is locally finite, we have for some integer $s$ the relation $L^{i} \subseteq \sum_{i=1}^{s} L^{i}$,

${ }^{23}$ ) This is Jacobson's Theorem 15 [6]. The proof given here is more in line with the rest of the section. 
$j=1,2, \cdots ;$ successive left multiplication of (22) yields

$$
M^{j} \subseteq \sum_{\rho=0}^{r} \sum_{\sigma=0}^{s} M^{\rho} L^{\sigma}, \quad j=1,2, \cdots: \rho+\sigma \geqq 1 .
$$

This shows that the algebra $\{M\}_{\Phi}$ is finite, q.e.d.

As a consequence of Lemma 6.4 we obtain:

Theorem 6.3. If $K$ is the locally finite kernel of an algebra $A$, then the locally finite kernel of the difference algebra $A-K$ is equal to 0.

CoRollary. The locally finite kernel $K$ of an algebra $A$ contains the maximal semi-nilpotent $\left({ }^{24}\right)$ ideal of the algebra. The difference algebra $A-K$ has no semi-nilpotent one-sided or two-sided ideals other than zero.

By [8] (or [13]) we know that any nil subring of a ring with finite index or with a polynomial identity is locally finite. Hence we have:

THEOREм 6.4. If an algebra $A$ has finite index, or if it satisfies polynomial identity, then the locally finite kernel $K$ of $A$ contains all right and left nil ideals of $A$. The algebra $A-K$ has no one-sided nonzero nil-ideals.

The radical of an algebraic algebra is a nil ideal. Hence we have:

CoRollary. If an algebraic algebra $A$ has finite index, or if it satisfies a polynomial identity, then the locally finite kernel $K$ of $A$ contains the radical of $A$, and the algebra $A-K$ is semi-simple.

7. Kurosch's problem. The notion of the locally finite kernel, combined with some of the structural results of the previous sections, leads to simplified, purely algebraic proofs of Kaplansky's results concerning the problem of Kurosch ${ }^{(25)}$.

Lemma 7.1. A plain, finitely generated algebraic algebra $A^{*}$ that satisfies a polynomial identity is locally finite.

Proof. We have to show that $A^{*}$ coincides with its locally finite kernel $K^{*}$. Suppose, on the contrary, that $A=A^{*}-K^{*} \neq 0$. Then $A$ satisfies the same conditions as $A^{*}$, and in addition its locally finite kernel is equal to 0 . In view of Theorem 6.3, the required contradiction will be established if we show that $A$ contains a nonzero locally finite ideal. To this end denote by $g_{1}, g_{2}, \cdots, g_{n}$ the generators of $A$ over the reference field $\Phi$, and consider a primitive ideal $P$ of $A$. Since along with $A$ also $A-P$ satisfies a polynomial identity, it is finite over its center (compare [8]), and on account of being

${ }^{(24)}$ For the definition and properties of the notion of semi-nilpotence compare [12]. For nil algebras semi-nilpotence and local finiteness are equivalent notions.

${ }^{(25)}$ The problem of Kurosch has been formulated by Jacobson [6] as follows: Is any algebraic algebra locally finite? 
finitely generated over $\Phi$, it follows that $A-P$ has a finite basis over $\Phi$. Denote by $b_{1}, b_{2}, \cdots, b_{r}$ a basis over $\Phi$ of $A$ modulo $P$. Then we have

$$
\begin{array}{rlrl}
g_{j} & =\sum_{i=1}^{r} \alpha_{i} b_{i}+c_{j} ; & c_{j} \in P, \alpha_{i} \in \Phi, \\
b_{j} b_{k}=\sum_{i=1}^{r} \alpha_{i j k} b_{i}+c_{j k} ; & c_{j k} \in P, \alpha_{i j k} \in \Phi .
\end{array}
$$

Any element $a$ of $A$ is a polynomial in the $g$ 's with coefficients in $\Phi$. Hence if $P^{*}$ denotes the ideal generated in $A$ by the finite set $c_{j}, j=1, \cdots, n, c_{j k}$, $j, k=1, \cdots, r$, it follows by (23) that $a=\sum_{i=1}^{n} \beta_{i} b_{i}+c, c \in P^{*}, \beta_{i} \in \Phi$. In particular, if $a \in P$, we have $\beta_{i}=0, i=1, \cdots, n$, or $a \in P^{*}$, i.e., $P=P^{*}$. Thus $P$ is a finitely generated ideal, which implies (e.g., by Theorem $5.5 \mathrm{~d}$ ) that $P$ has a unit. It follows therefore that $A=B \oplus P, B \cong A-P$, which shows that $A$ contains a nonzero locally finite ideal $B$. This is the required contradiction.

THEOREM 7.1. Any algebraic algebra $A$ that satisfies a polynomial identity is locally finite $\left({ }^{26}\right)$.

Proof. As in the proof of the preceding lemma, it suffices to show that if the locally finite kernel of the algebra $A$ is equal to 0 , then also $A=0$. Indeed, suppose that $A \neq 0$, then by the corollary of Theorem 6.4 we know that $A$ is semi-simple, and by Theorem 3.3 that $A$ contains a matrix ideal $M_{0}$. Now $M_{0} \cong B_{m}$, where $B_{m}$ is a total matrix ring of some finite degree $m$, over a plain algebra $B$. By Lemma 7.1 we know that $B$ is locally finite. Hence also $M_{0}$ is locally finite, which is impossible since $A$ has no nonzero locally finite ideals (Theorem 6.3). This contradiction completes the proof.

Theorem 7.2. Let $A$ be an algebraic algebra, and suppose that: (1) Any primitive image of $A$ satisfies a polynomial identity. (2) Any nil ideal of any homomorphic image of $A$ is locally finite. Then $A$ is locally finite $\left({ }^{27}\right)$.

Proof. If $K(A)$ is the locally finite kernel of $A$, then by condition (2) it follows in view of Theorem 6.3 that the algebra $A^{*}=A-K(A)$ is either equal to 0 or semisimple. Since along with $A$ also $A^{*}$ satisfies condition (1) of the theorem, it follows by Theorem 3.5 in case $A^{*} \neq 0$ that $A^{*}$ contains a matrix ideal that satisfies a polynomial identity, which as in the proof of Theorem 7.1 leads to a contradiction. Hence $A^{*}=0$, i.e., $A=K(A)$, q.e.d.

(26) This is Kaplansky's Theorem 6.1 [9].

(27) Condition (2) of this theorem seems to be indispensable also in Kaplansky's proof of the same theorem (compare [10, Theorem 10.3]). Indeed, in case $\rho$ is a limit ordinal, the quotient rings $A / I_{\rho}$ that appear in his proof may contain nil ideals of a rather general type. However, this alteration does not affect the validity of his Theorems 10.4 and 10.5 which are based on Theorem 10.3. 
One readily obtains the following:

Corollary 1. If any primitive image of an algebraic algebra $A$ with finite index satisfies a polynomial identity, then $A$ is locally finite.

As a consequence of Theorem 5.4 it follows that Corollary 1 may be regarded as a special case of the following:

COROLlary 2. If every primitive image of an algebraic algebra $A$, as well as every nil ideal of any homomorphic image of $A$, satisfy polynomial identities, then $A$ is locally finite.

\section{BIBLIOGRAPHY}

1. A. S. Amitsur and J. Levitzki, Minimal identities for algebras, Proceedings of the American Mathematical Society vol. 1 (1950) pp. 449-463.

2. R. Arens and I. Kaplansky, Topological representation of algebras, Trans. Amer. Math. Soc. vol. 63 (1948) pp. 457-481.

3. R. Baer, Radical ideals, Amer. J. Math. vol. 65 (1943) pp. 537-568.

4. A. Forsyth and N. H. McCoy, The commutativity of certain rings, Bull. Amer. Math. Soc. vol. 52 (1946) pp. 523-526.

5. N. Jacobson, The radical and semi-simplicity for arbitrary rings, Amer. J. Math. vol. 61 (1945) pp. 300-320.

6. - Structure theory for algebraic algebras of bounded degree, Ann. of Math. vol. 46 (1945) pp. 695-707.

7. - Some remarks on one-sided inverses, Proceedings of the American Mathematical Society vol. 1 (1950) pp. 352-355.

8. I. Kaplansky, Rings with a polynomial identity, Bull. Amer. Math. Soc. vol. 54 (1948) pp. $575-580$.

9. - Topological representation of algebras. II, Trans. Amer. Math. Soc. vol. 68 (1950) pp. 62-75.

10. - The structure of certain operator algebras, Trans. Amer. Math. Soc. vol. 70 (1951) pp. 219-255.

11. G. Koethe, Die Struktur der Ringe deren Restklassenring nach dem Radikal vollstaendig reduzibel ist, Math. Zeit. vol. 32 (1930) pp. 161-186.

12. J. Levitzki, On the radical of a general ring, Bull. Amer. Math. Soc. vol. 49 (1943) pp. $462-466$.

13. - A theorem on polynomial identities, Proceedings of the American Mathematical Society vol. 1 (1950) pp. 334-341.

14. N. McCoy, Generalized regular rings, Bull. Amer. Math. Soc. vol. 45 (1939) pp. 175-178.

UNIVERSITY OF JERUSALEM, JERUSALEM, ISRAEL. 